\title{
Numerical simulation of powder transport behavior in laser cladding with coaxial powder feeding
}

\author{
LIU Hao ${ }^{1,2^{*}}, \mathrm{HE} \mathrm{XiuLi}^{3}$, YU Gang $^{3 *}$, WANG ZhongBin ${ }^{1}$, LI ShaoXia ${ }^{3}$, \\ ZHENG CaiYun ${ }^{3}$ \& NING WeiJian ${ }^{3}$ \\ ${ }^{1}$ School of Mechanical and Electrical Engineering, China University of Mining and Technology, Xuzhou 221116, China; \\ ${ }^{2}$ Jiangsu Key Laboratory of Mine Mechanical and Electrical Equipment, China University of Mining and Technology, Xuzhou 221116, China; \\ ${ }^{3}$ Key Laboratory of Mechanics in Advanced Manufacturing, Institute of Mechanics, Chinese Academy of Sciences, Beijing 100190, China
}

Received March 11, 2015; accepted June 2, 2015

\begin{abstract}
Laser cladding with coaxial powder feeding is one of the new processes applied to produce well bonding coating on the component to improve performance of its surface. In the process, the clad material is transported by the carrying gas through the coaxial nozzle, generating gas-powder flow. The powder feeding process in the coaxial laser cladding has important influence on the clad qualities. A 3D numerical model was developed to study the powder stream structure of a coaxial feeding nozzle. The predicted powder stream structure was well agreed with the experimental one. The validated model was used to explore the collision behavior of particles in the coaxial nozzle, as well as powder concentration distribution. It was found that the particle diameter and restitution coefficient greatly affect the velocity vector at outlet of nozzle due to the collisions, as well as the powder stream convergence characteristics below the nozzle. The results indicated a practical approach to optimize the powder stream for the coaxial laser cladding.
\end{abstract}

laser cladding, solid-gas flow, numerical simulation, collision behavior

PACS number(s): 47.55.Kf, 45.50.Tn, 42.62.Cf, 02.70.Bf, 83.50.Ha

Citation: Liu H, He X L, Yu G, et al. Numerical simulation of powder transport behavior in laser cladding with coaxial powder feeding. Sci China-Phys Mech Astron, 2015, 58: 104701, doi: 10.1007/s11433-015-5705-4

\section{Introduction}

Laser cladding is an advanced process to produce clad layer on the substrate using high density laser beam. Laser cladding can also be used to repair damaged parts and even to fabricate complete parts by multi-layer deposition. Compared with conventional coating process such as thermal spraying, laser cladding has many advantages, including minimal dilution and distortion, higher bond strength between coating and substrate, better surface quality, better process flexibility [1-4].

In laser cladding, the powder is delivered laterally or co-

*Corresponding authors (LIU Hao, email: liuhao56@cumt.edu.cn; YU Gang, email: gyu@imech.ac.cn )

๑) Science China Press and Springer-Verlag Berlin Heidelberg 2015 axially into the melt pool by inert gas flow. The melt pool solidified rapidly when the laser beam moves away, then a thin layer is obtained on the substrate. The width of the layer of single laser track is limited by the laser beam diameter, so overlapping of laser tracks is necessary to produce large area coating on the component surface [4,5]. Thus, coaxial powder feeding is more suitable than lateral powder feeding, since it offers precise and equal feeding rate in any direction $[4,6]$.

The coaxial laser cladding process can be broadly divided into two stages. In the first stage, the powder is transported into the substrate by gas flow. Laser beam, powder flow, and gas flow interact with each other before powder particles reach the melt pool. The laser energy is absorbed

phys.scichina.com link.springer.com 
partly by the powder stream, heating the in-flight particles to melt. In the second stage, the melt pool solidifies rapidly and a thin layer of material is deposited on the substrate after the laser beam moved away. The powder transport behaviors in the first stage of coaxial laser cladding not only dominate the mass of powder particles reached the melt pool and the thermal status of those powder particles, but also determine the laser power attenuation due to the material-radiation interaction [7]. It is essential to study the powder stream characteristics in order to build the coating with accurate dimensions and improve powder using efficiency [8].

Considering many variables such as the structure and size of coaxial nozzle, gas flow rate and powder feeding rate associated with powder stream, experimental investigations to explore the correlations between main variables and powder stream characteristics are rather expensive and time-consuming. In recent years, several analytical and numerical modeling efforts have been reported to provide insights into powder transport process during coaxial laser cladding and predict the powder concentration distribution. Pinkerton [6,7] developed a mathematical model for the powder concentration distribution in laser-based rapid tooling and demonstrated its application to evaluation of nozzle geometry and calculation of laser beam attenuation. Yang et al. [9] proposed a Gaussian concentration model to simulate the volume fraction distribution of powder particle stream in coaxial cladding. It was found that smaller exit width of the coaxial nozzle gave smaller powder stream diameter and higher peak powder concentration at focus position. In these analytical models, the interaction between powder particles and gas flow was neglected, the shape of powder stream mainly depended on the coaxial nozzle geometry. In addition, Gaussian powder distribution was assumed in most analytical models. Consequently, more numerical models were developed based on gas-solid two phase flow theory. By solving the coupled momentum transfer equations between the powder particles and gas flow phase, the dynamic behavior of powder particles in gas flow could be modeled completely. Lin et al. [10] developed a 2D numerical model and studied the focused and columnar powder streams of a coaxial nozzle with various arrangements of the nozzle exit and gas flow settings. Zhu et al. [11] also developed a 2D numerical model to simulate the influencing rule of deposited layer's shape on coaxial powder feeding flow field in laser direct metal deposition. The whole continuous coaxial nozzle is not axial symmetry taking the powder inlet into account, and gas flow is turbulent flow which in turn is a 3D phenomenon. Hence, 2D model is deemed not suitable to investigate flight behavior of particles in the nozzle comparing with 3D model. Zekovic et al. [12] modeled 3D multi-phase gas-powder flow structures of coaxial nozzles using computational fluid dynamics methods. The reliability of 3D model was evaluated by comparing the calculated results with the experimental results. The analysis based on results proved the importance of the secondary gas flow for protecting laser head optics by blocking ricocheted particles. Tabernero et al. [13] established a 3D numerical model to predict the powder flux distribution on a coaxial nozzle. The model was validated by the experiments measuring powder flux distribution for three different kinds of metal powder. They investigated the powder flux distribution evolution from an annular profile to a Gaussian profile by the numerical simulation and experiments. Wen et al. [14] presented a systematic model to estimate the dynamic behavior of coaxial powder flow as well as particle temperatures for direct deposition processes, but effects of substrate on the powder flow were ignored in this model. Balu et al. [15] developed a 3D computational fluid dynamics model to characterize the coaxial powder flow behavior. Their work indicated that powder flow characteristics were affected by the morphology and physical properties of powder.

Majority of the above powder stream models have neglected the collisions between particles and the internal wall of coaxial nozzle. Often, the directions of all powder particles were assumed parallel to the nozzle axis in all these simulations, thereby imposing an unrealistic initial condition. Despite numerous studies of the powder stream phenomenon of the coaxial laser cladding, they mainly focused on powder concentration distribution evolution based on a given coaxial nozzle and gas flow settings [11-14], as well as structure optimization of coaxial nozzle to make powder stream converged $[6,7,10]$. The particle collision behavior and its effect on the powder stream structure were barely discussed in those works.

In this paper, a 3D numerical model is developed based on a given coaxial nozzle considering the particle collision behavior in the nozzle. The model is validated by comparing the simulated and experimental powder stream structure and utilized to calculate the particle trajectories and powder concentration distribution. Based on the analysis of particle collision and trajectory, two key factors, namely particle diameter and restitution coefficient, and their effect on the powder stream characteristics are discussed further.

\section{Powder stream modeling}

\subsection{Description and assumptions}

The powder transport process is shown in Figure 1. There are three different kinds of gas flow in the coaxial nozzle. At the center, the inner shielding gas flow is parallel to the laser beam and its main purpose is to protect the lenses from the hot powder particles that ricocheted from the substrate. The carrying gas flow is to deliver the powder particles to the melt pool. The outer shielding gas flow generates protective atmosphere in peripheral of powder flow to prevent powder particles from oxidation. All these flows and their interactions affect the powder concentration distribution 


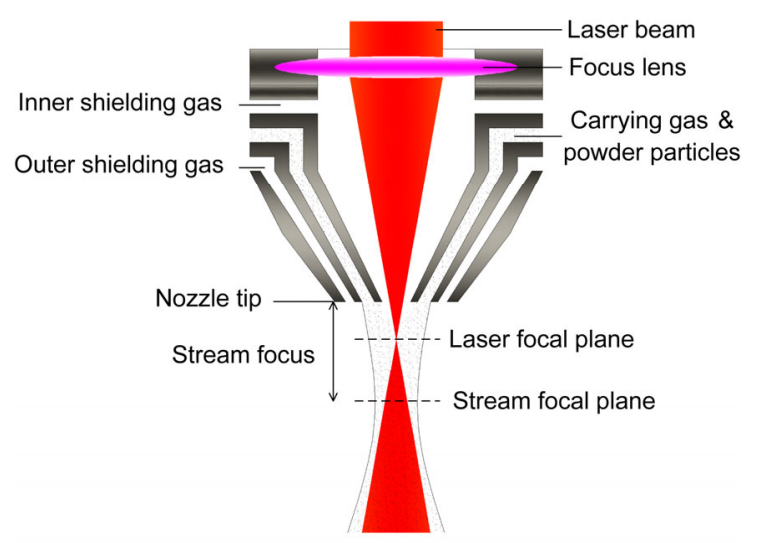

Figure 1 (Color online) Schematic diagram of powder transporting process in coaxial laser cladding.

below the exit of coaxial nozzle.

Actually the powder stream is solid-gas two-phase flow. The shielding and carrying gas should be considered as the continuous phase while powder particles acts as a discrete phase into the continuous phase in the powder stream. Therefore, the model of powder stream consists of two different modules. The proposed model is based on the following assumptions [12].

(1) The gas flow is treated as a steady-state turbulent flow with constant velocity distribution in the inlet boundary. At the shielding gas and gas-powder inlet, the velocity of gas flow and particles is considered constant and perpendicular to the inlet surface of the nozzle. This is reasonable given the long time the gas takes to go through the gas pipe between powder feeder and the coaxial nozzle.

(2) The forces of drag, inertia and gravity are considered in the model, while other forces such as pressure and surrounding flow acceleration are neglected.

(3) Since the mean volume fraction of particles is less than $10 \%$, the probability of powder particles collision is very low. In this case, the particle collision has little influence on the powder concentration distribution. So the interaction between particles is not considered to simplify the modeling and reduce the calculation time.

(4) The particle size is assumed to follow the general Rossin-Rammler distribution expression.

(5) Heat transfer by laser radiation is not included because the powder flow is not influenced by laser beam.

(6) Powder particles flow represented as a discrete phase does not affect the continuous phase due to the low mass and concentration of the particles.

\subsection{Continuous phase modeling}

The continuous phase modeling is based on Navier-Stokes differential equations with the Reynolds method of averaging time-dependent equations, together with the standard $k-\varepsilon$ turbulent model. Since the gas flow is steady, incompressi- ble, isothermal and chemically homogeneous, the equations for turbulent flow are expressed as follows.

Conservation of mass

$$
\frac{\partial}{\partial x_{i}}\left(\rho u_{i}\right)=0
$$

where $\rho$ is gas density, and $u_{i}$ is the velocity vector in the $i$ th direction.

Conservation of momentum:

$$
\frac{\partial}{\partial x_{j}}\left(\rho u_{i} u_{j}\right)=-\frac{\partial p}{\partial x_{i}}+\frac{\partial \tau_{i j}}{\partial x_{j}}+\rho g_{i},
$$

where $p$ is pressure, $g_{i}$ is gravitational acceleration and $\tau_{i j}$ is the viscous stress given by

$$
\tau_{i j}=\left[\left(\mu+\mu_{\mathrm{t}}\right)\left(\frac{\partial u_{i}}{\partial x_{j}}+\frac{\partial u_{j}}{\partial x_{i}}\right)\right]-\frac{2}{3} \mu_{\mathrm{t}} \frac{\partial u_{i}}{\partial x_{i}} \delta_{i j},
$$

where $\mu$ is the molecular viscosity. $\delta_{i j}=1$ for $i=j$, otherwise $\delta_{i j}=0$, and $\mu_{\mathrm{t}}$ is the turbulent viscosity defined by

$$
\mu_{\mathrm{t}}=\rho C_{\mu} \frac{k^{2}}{\varepsilon},
$$

where $C_{\mu}=0.09$ is a constant, $k$ is the kinetic energy of turbulence, and $\varepsilon$ is the dissipation of kinetic energy of turbulence. The standard turbulent $k$ - $\varepsilon$ model is adopted to solve these equations.

Conservation of kinetic energy of turbulence:

$$
\frac{\partial}{\partial x_{i}}\left(\rho u_{i} k\right)=\frac{\partial}{\partial x_{i}}\left(\frac{\mu_{\mathrm{t}}}{\sigma_{\mathrm{k}}} \frac{\partial k}{\partial x_{i}}\right)+G_{\mathrm{k}}+G_{\mathrm{b}}-\rho \varepsilon .
$$

Conservation of dissipation of kinetic energy of turbulence:

$$
\begin{gathered}
\frac{\partial}{\partial x_{i}}\left(\rho u_{i} \varepsilon\right)=\frac{\partial}{\partial x_{i}}\left(\frac{\mu_{\mathrm{t}}}{\sigma_{\varepsilon}} \frac{\partial \varepsilon}{\partial x_{i}}\right)+C_{1 \varepsilon} \frac{\varepsilon}{k}\left(G_{\mathrm{k}}+G_{\mathrm{b}}\right)-C_{2 \varepsilon} \rho \frac{\varepsilon^{2}}{k} \\
G_{\mathrm{k}}=\mu_{\mathrm{t}}\left(\frac{\partial u_{j}}{\partial x_{i}}+\frac{\partial u_{i}}{\partial x_{j}}\right) \frac{\partial u_{i}}{\partial x_{j}}, \\
G_{\mathrm{b}}=-g_{i} \frac{u_{\mathrm{t}}}{\rho P r_{\mathrm{t}}} \frac{\partial \rho}{\partial x_{i}}
\end{gathered}
$$

where $C_{1 \varepsilon}=1.44, C_{2 \varepsilon}=1.92, \sigma_{\mathrm{k}}=1.0$ and $\sigma_{\varepsilon}=1.3$ are empirical constants, $P r_{\mathrm{t}}$ is the turbulent Prandtl number, $G_{\mathrm{k}}$ is the rate production of turbulence kinetic energy owing to the mean velocity gradients, $G_{\mathrm{b}}$ is the generation of turbulence kinetic energy due to buoyancy [13].

\subsection{Discrete phase modeling}

The gas-powder flow is a typical two phase flow problem considering powder particles as the second phase. It can be 
solved with the additional transport equations of the second phase. The trajectory of each powder particle is described in a Lagrangian reference frame based on a force balance. For a particle of density $\rho_{P}$ and diameter $d_{P}$, This force balance can be written as:

$$
\frac{\mathrm{d} u_{P i}}{\mathrm{~d} t}=F_{\mathrm{D}}\left(u_{i}-u_{P i}\right)+\frac{\left(\rho_{P}-\rho\right) g_{i}}{\rho_{P}}+F_{i},
$$

where $u_{P i}$ is the velocity of particle, $u_{i}$ is the velocity of the gas flow, $F_{\mathrm{D}}\left(u_{i}-u_{P i}\right)$ is the drag force per unit particle mass, and $F_{i}$ is additional forces.

$$
F_{\mathrm{D}}=\frac{18 \mu}{\rho_{P} d_{P}^{2}} \frac{C_{\mathrm{D}} R e}{24}
$$

where $R e$ is relative Reynolds number given by

$$
R e=\frac{\rho d_{P}\left|u_{i}-u_{P_{i}}\right|}{\mu},
$$

and $C_{\mathrm{D}}$ is the drag coefficient, and it is a function of the relative Reynolds number and shape factor for non-spherical particles [16]:

$$
\begin{gathered}
C_{\mathrm{D}}=\frac{24}{R e}\left(1+a_{1} \operatorname{Re} e^{a_{2}}\right)+\frac{a_{3} R e}{a_{4}+\operatorname{Re}}, \\
a_{1}=\exp \left(2.3288-6.4581 \varphi+2.4486 \varphi^{2}\right), \\
a_{2}=0.0964+0.05565 \varphi, \\
a_{3}=\exp \left(4.905-13.8944 \varphi+18.4222 \varphi^{2}-10.2599 \varphi^{3}\right), \\
a_{4}=\exp \left(1.4681+12.2584 \varphi-20.7322 \varphi^{2}+15.8855 \varphi^{3}\right),
\end{gathered}
$$

where $\varphi$ is shape factor of particle. For actual metal particles are not perfectly spherical, shape factor is defined to describe how spherical a particle is and expressed as:

$$
\varphi=\frac{s}{S}
$$

where $s$ is the surface area of a sphere with the same volume as the particle, and $S$ is the actual surface area of the particle. When the particle is exactly sphere, the shape factor equals one. The smaller the shape factor is, the less spherical the particle is.

The size distribution of the powder is described by Rosin-Rammler distribution. This method is to divide the whole range of particle sizes into several numbers of discrete intervals. Each interval is represented by a mean diameter $d$ for trajectory calculations, and the mass fraction of particles with diameter greater than $d$ is expressed by

$$
F_{d}=\exp \left(-\left(\frac{d}{d_{m}}\right)^{n}\right)
$$

where $d_{m}$ is the size constant, and $n$ is the spread parameter.

With the time integral of the force balance differential equation, the velocity of the particle at each point along the trajectory is acquired. The particle trajectory can be predicted by the kinematic equation as follow:

$$
\frac{\mathrm{d} x_{i}}{\mathrm{~d} t}=u_{p i}
$$

where $x_{i}$ represents the position of the particle.

The momentum loss of particle collision with internal wall of nozzle is evaluated by the restitution coefficient, which is defined as the ratio of velocities before and after collision:

$$
e_{n}=\frac{u_{2 p}}{u_{1 p}}
$$

where subscript of 1 and 2 means before collision and after collision. The $e_{n}$ of elastic collision is 1 , meaning the momentum is not loss in the collision, and the $e_{n}$ of inelastic collision is less than 1 . In general, restitution coefficient is a function of the powder and nozzle material, impact velocity, hardness ratio, nozzle wall roughness, and it is in the range from 0.9 to 0.99 [17]. The $e_{n}$ is considered as constant when the material of coaxial nozzle and powder are selected in this paper.

\section{Model application}

The solution technique used in this study is based on the FLUENT software, which solves the governing equations of continuous phase and discrete phase listed above by a specified finite-volume method.

The structure of coaxial nozzle used for modeling is shown in Figure 2. The coaxial nozzle has four gas inlets, connecting with an annular channel. The inner shielding gas inlet is located above the exit of the nozzle, and two outer shielding gas inlets connect with the outer annular channel. The main structural parameters of the coaxial nozzle are shown in Table 1 . The intersection point of the middle passage lines is located $7 \mathrm{~mm}$ below the nozzle tip according to the structural parameters.

As is shown in Figure 3, the meshed geometry used in the model consists of two parts: one represents the cavity of the coaxial nozzle; the other is computational domain below
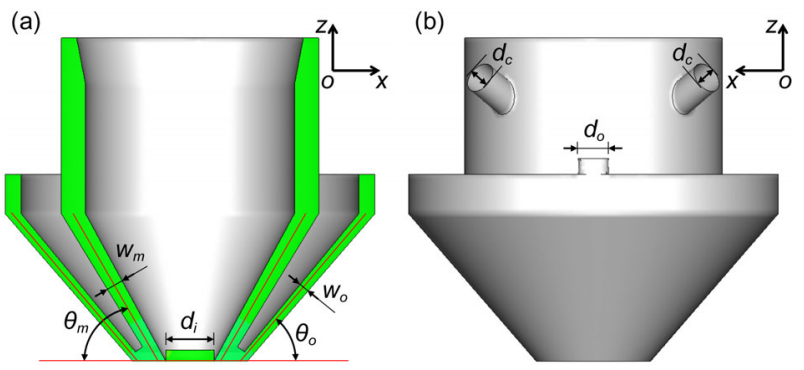

Figure 2 (Color online) The structure of coaxial nozzle used for modeling: (a) symmetrical section; (b) front view. 
Table 1 Main structural parameters of the coaxial nozzle

\begin{tabular}{ccc}
\hline Variable & Symbol & Value \\
\hline Inner gas inlet diameter & $d_{i}$ & $6 \mathrm{~mm}$ \\
Carrying gas inlet diameter & $d_{c}$ & $4 \mathrm{~mm}$ \\
Outer gas inlet diameter & $d_{o}$ & $2 \mathrm{~mm}$ \\
Middle annular passage width & $w_{m}$ & $2.2 \mathrm{~mm}$ \\
Middle annular passage angle & $\theta_{m}$ & $60^{\circ}$ \\
Outer annular passage width & $w_{o}$ & $1.2 \mathrm{~mm}$ \\
Outer annular passage angle & $\theta_{o}$ & $50^{\circ}$ \\
\hline
\end{tabular}

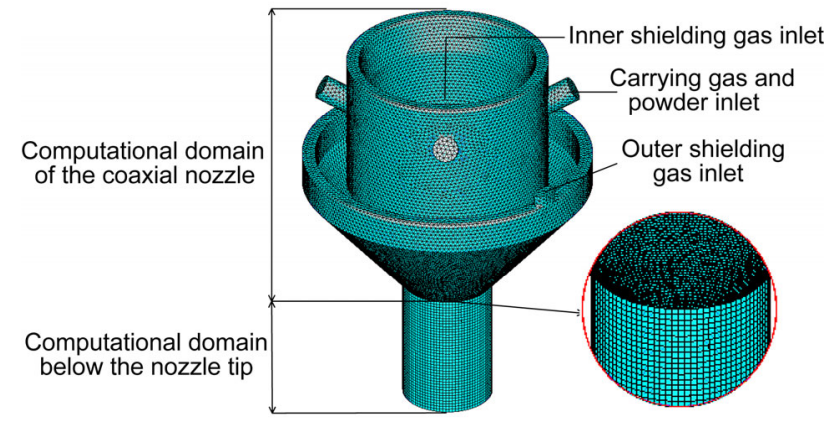

Figure 3 (Color online) The meshed geometry for the powder stream calculations.

the nozzle tips. The body fitted coordinate (BFC) grid system is applied, which allowed the non-standard geometry of the nozzle to be mapped into Cartesian or cylindrical geometry. The coaxial nozzle part of the model is meshed with unstructured tetrahedral and hexahedral elements due to the complexity of geometry, and the other part is meshed with structured hexahedral elements for the accuracy of calculation.

All the inlets are considered as velocity inlets for the continuous phase. Metal powder is delivered by gas flow at a carrying gas velocity $u_{c}$. The inner shielding gas flows from the inlet at a velocity $u_{i}$, and outer shielding gas at a velocity $u_{o}$. The computational domain of coaxial nozzle is bounded by the wall boundary condition. As a result, the rebounds of particle within the internal wall can be predicted by the model. The cylindrical space below the nozzle, which has $20 \mathrm{~mm}$ in height and $14.2 \mathrm{~mm}$ in diameter, is large enough to capture sufficient particle trajectories. The side and bottom surface of this part are defined as pressure outlet boundary conditions, the particles escape from the computational domain when they reach these boundaries.

Experiment was carried out using NiCoCrAlY powder to validate the model. Argon was used as carrying and shielding gas in the experiment. The NiCoCrAlY powder has extensive applications in aerospace industry to produce thermal barrier coating. The morphology of the powder was examined by a scanning electron microscope (SEM) as is shown in Figure 4.

It can be seen that most of the powder particles are near spherical or ellipsoidal in shape. Based on the SEM micro-

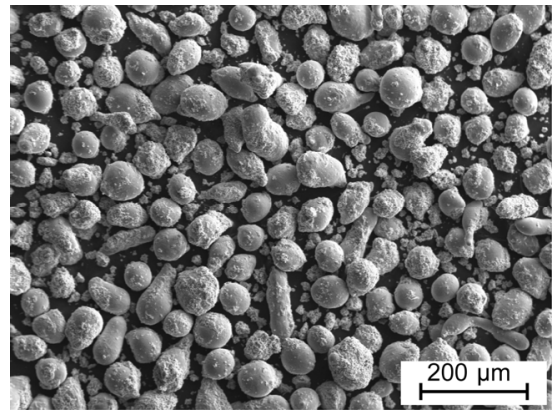

Figure 4 NiCoCrAlY powder particle morphology.

graphs, a value of 0.9 was chosen as the shape factor for the powder used. The particle size distribution of the powder was measured by sieve analysis and approximated by Rosin-Rammler distribution. The minimum and maximum diameters were chosen to be $45 \mu \mathrm{m}$ and $130 \mu \mathrm{m}$, and this range was divided into 9 equal intervals. The spread parameter $n$ of 4.7 was obtained based to eq. (15). Figure 5 shows the comparison of measured and assumed size distribution of the powder.

To associate the experimental process parameters with numerical computation, the following equation has been used to describe the relationship between gas velocity and volume according to the nozzle structure:

$$
u=\frac{Q}{S}
$$

where $u$ is the gas velocity at nozzle inlet, $Q$ is the gas volume per unit time, and $S$ is the sectional area of the nozzle inlet.

\section{Results and discussion}

\subsection{Model and experiment comparison}

An experiment was performed to validate the calculated

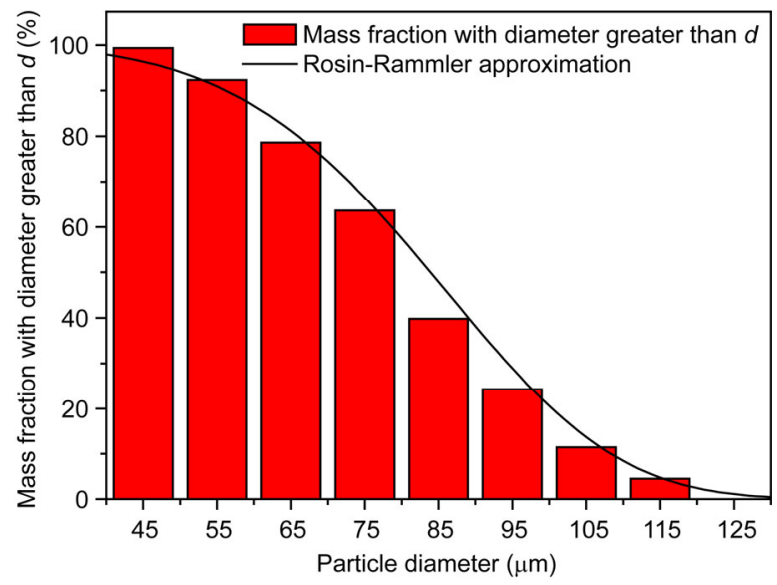

Figure 5 (Color online) The comparsion of measured and assumed particle size distribution of NiCoCrAlY powder. 
results. During the experiment, the coaxial nozzle was placed in a camera black box with a slit in the side. A halogen lamp light was used to illuminate the powder stream though the slit. The front view of powder stream can be imaged by the camera with an appropriate exposure time. Because powder particles reflect the light, the gray level of the powder stream image can be considered proportional to the powder particle quantity in the local region [15]. The powder feeding parameters used were: $8.5 \mathrm{~L} / \mathrm{min}$ of inner shielding gas, $3 \mathrm{~L} / \mathrm{min}$ of carrying gas, $4 \mathrm{~L} / \mathrm{min}$ of outer shielding gas and $4.2 \mathrm{~g} / \mathrm{min}$ of powder feed rate. The experimental image and calculated powder stream are shown in Figure 6. To reveal the flight behavior of multi-particles in the powder stream, a total of 129600 particle trajectories with different incident location in the section of inlets have been calculated in the case. Figure 6(b) describes only 216 of these particle trajectories from two powder inlets for the sake of clearness of the image. The color in the path line indicates the powder concentration in the local area. Figure 6 shows that the predicted powder stream structure below the coaxial nozzle tip is in reasonable agreement with the experimental measurement, which demonstrates that the model can be used further to study the powder transporting characteristics during the coaxial laser cladding.

It is observed that powder flow is annular when it is delivered out of the coaxial nozzle tip. The powder flow converges to a waist where the powder stream focuses to a high concentration zone. After the powder flow merged, it gradually diverges due to the inertia of particles. Three different zones can be divided by the powder concentration distribution of transverse planes: annular zone, consolidation zone and dispersed zone. The annular zone is just below the exit of nozzle, and the powder concentration in the center increases with the standoff distance increasing in this zone. The consolidation zone is where the powder concentration in the center highest in the horizontal plane. The dispersed zone where the powder particles disperse from the center is located below the consolidation zone.
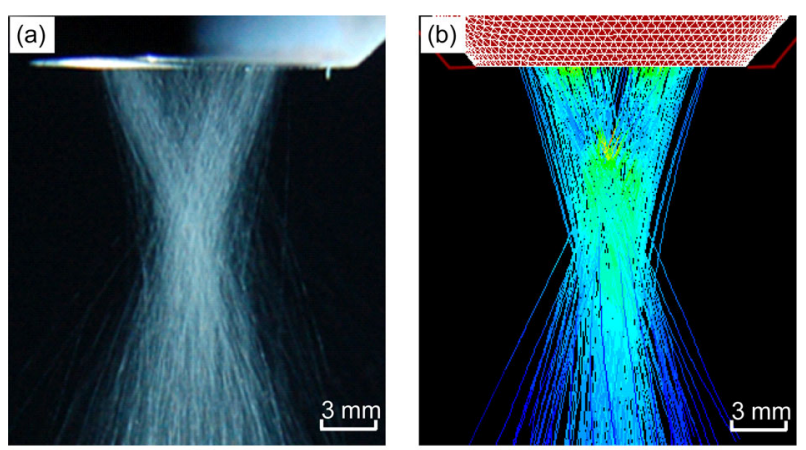

Powder concentration $\left(\mathrm{kg} / \mathrm{m}^{3}\right)$

11.522 .533 .544 .555 .566 .57

Figure 6 (Color online) Comparison of experimental and predicted powder stream structure: (a) experimental; (b) predicted.
To obtain more information about each zone, powder concentration along radial direction at different horizontal plane below the coaxial nozzle is shown in Figure 7 . The $\mathrm{Z}$ coordinate values of these planes are $0,2.5,5,7.9,12.5$ and $17.5 \mathrm{~mm}$, respectively. It is worth to note that powder concentration distribution in each horizontal plane is approximately symmetrical about the nozzle axis $(x=0 \mathrm{~mm}, y=0$ $\mathrm{mm}$ ), which is the reason that coaxial laser cladding is independent from motion direction. At the plane of $z=0 \mathrm{~mm}$, annular peak concentration reaches about $3.0 \mathrm{~kg} / \mathrm{m}^{3}$, while in the center the powder concentration is near zero. The annular peak concentration decreases when the distance from the nozzle tip increases. At the same time, powder concentration in the center becomes higher until the focal plane at $z=7.9 \mathrm{~mm}$, which indicates that the powder stream is converged.

It is observed that at the focal plane the powder concentration decreases rapidly from $3.5 \mathrm{~kg} / \mathrm{m}^{3}$ to $1.0 \mathrm{~kg} / \mathrm{m}^{3}$ within $2 \mathrm{~mm}$ of the nozzle axis, and the downward trend slows when it is $2 \mathrm{~mm}$ to $3 \mathrm{~mm}$ away from nozzle axis. The length from the center to the stable region of powder concentration can be defined as the effective powder convergence radius. It can be concluded that the laser beam radius on the substrate surface should be approximately equal to this radius for a desirable deposition. The consolidation zone is in the range of $z=5-12.5 \mathrm{~mm}$. It is ideal deposition zone where the substrate should be placed for a high powder using
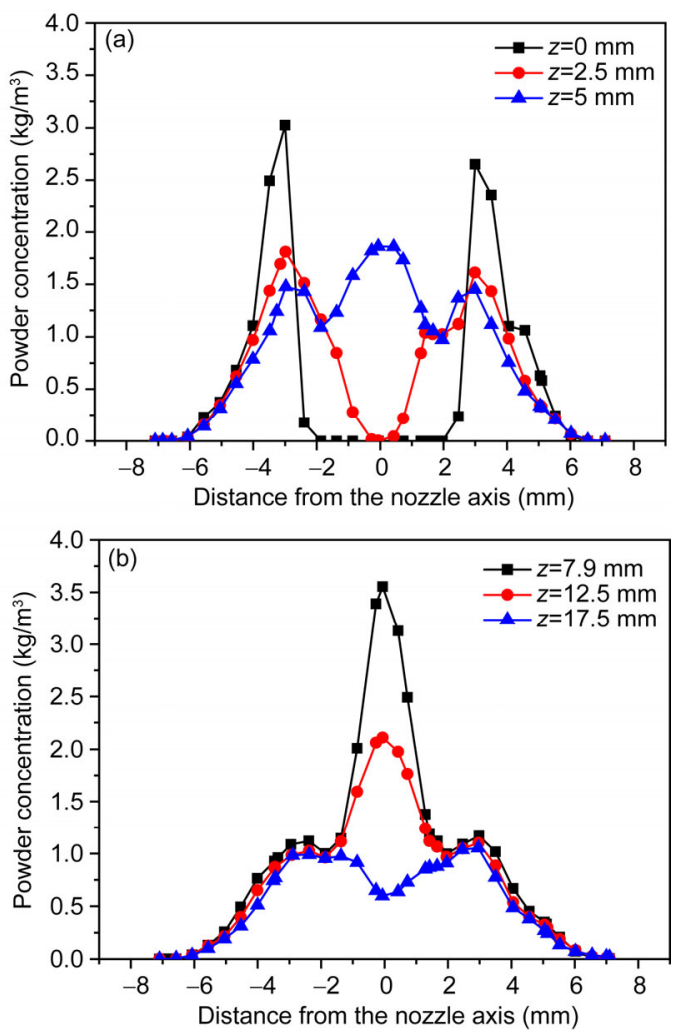

Figure 7 (Color online) Powder concentration distribution along radial direction at different transverse planes below the coaxial nozzle. 
efficiency.

When the gas flow rates are constants, curves of powder concentration along the nozzle axis with different powder feed rates are plotted in Figure 8. The peak powder concentration increases from $1.55 \mathrm{~kg} / \mathrm{m}^{3}$ to $5.43 \mathrm{~kg} / \mathrm{m}^{3}$ with the powder feed rate varying from $1.75 \mathrm{~g} / \mathrm{min}$ to $6.55 \mathrm{~g} / \mathrm{min}$, but the focal plane of the powder stream keeps at $z=7.9 \mathrm{~mm}$. This result demonstrates that a proper combination of shielding gas flow and carrying gas flow has the ability to deliver powder in a certain feed rate range, which is meaningful to guarantee the stability of laser cladding process with varying powder feed rate.

\subsection{Collision between the nozzle walls}

Figure 9 shows the status of a powder particle at the coaxial nozzle tip. Taking a single particle as research object, the force acting on the particle beneath the coaxial nozzle is gravity and drag by the gas flow, and velocity vector can be taken as the initial condition of its trajectory below the nozzle. The gas flow mainly depends on the process parameters including $u_{i}, u_{o}$ and $u_{c}$, while velocity vector is strongly influenced by the collision behavior with the internal wall in

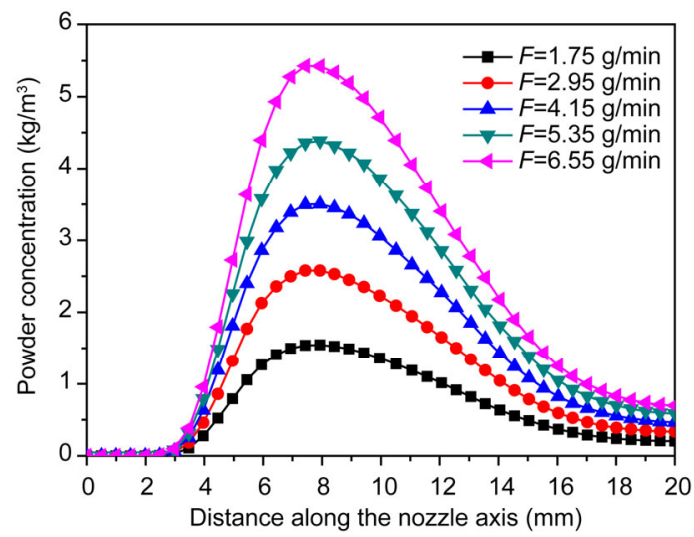

Figure 8 (Color online) Powder concentration along the nozzle axis on various powder feed rates when the gas flow rates are selected as constants.

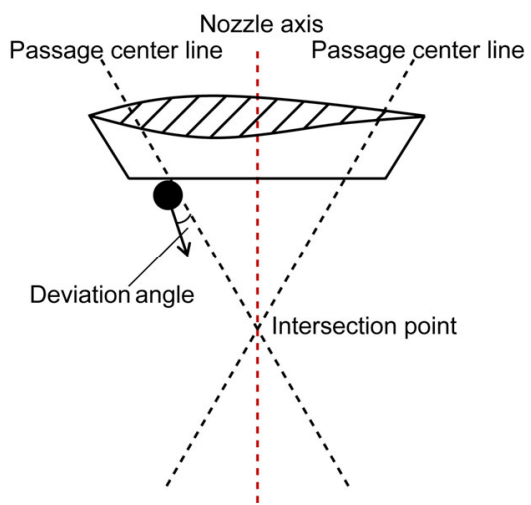

Figure 9 (Color online) Deviation angle between particle velocity at the nozzle tip and the passage center line. the coaxial nozzle. Generally, the velocity deviates from the annular passage after plenty of collisions. The statistical tendency of deviation angles due to the collision apparently affects the powder stream structure, for it is the consequence of a large number of powder particle trajectories.

Figure 10 presents the particles rebounding between the nozzle interior walls. Ten particle trajectories with different diameters starting at the same point of the nozzle are showed. It can be found that particles collide with internal wall of the coaxial nozzle many times, and the velocities of particles decrease until they come out of the nozzle tip. The velocities of particles are set to $1 \mathrm{~m} / \mathrm{s}$ based on the assumption. After plenty of collisions, the velocities with different particle diameters decrease to the range of $0.4-0.8 \mathrm{~m} / \mathrm{s}$. The trajectories are nearly consistent until the collision occurs three times. The location and the flight direction of particles are dispersed when the particles reach the horizontal plane of the nozzle tip. The scattered locations make the powder concentration distributed more uniform circumferentially, which is an advantage of continuous coaxial powder injection comparing with discontinuous coaxial powder injection.

Figure 11 represents velocity curves of particle trajectories with different diameters. It can be seen that each velocity curve is composed by two stages, one is in the coaxial nozzle that the collision leads to step change of particle velocity, the other is below the nozzle tip that the velocity increases continuously. In the coaxial nozzle, the momentum is in loss status in the nozzle under different particle diameters, for the velocity at nozzle tip is less than that at the powder inlet. This demonstrates that the collision plays a major role in the particle flight. When the particle gets out of the nozzle, its velocity rises rapidly under the action of gravity and the drag force of gas flow.

It can also be inferred from Figure 11 that the particle

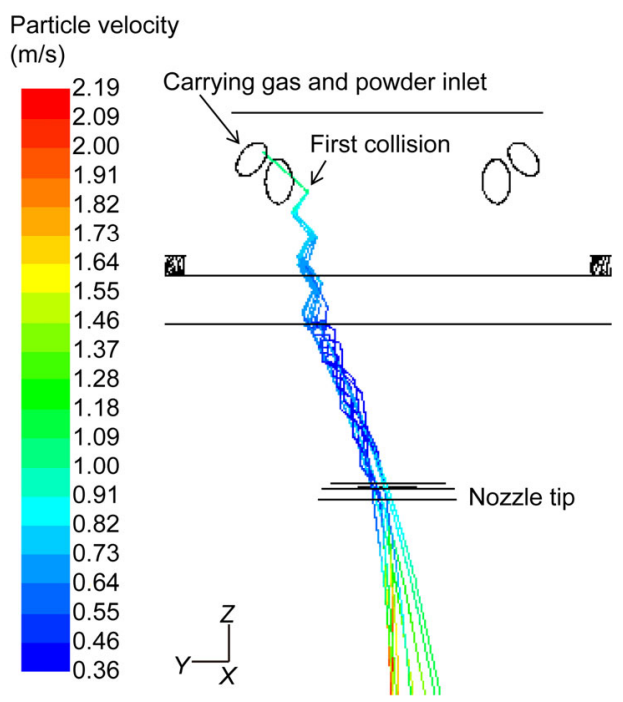

Figure 10 (Color online) The particles rebounding from the nozzle interior wall. 


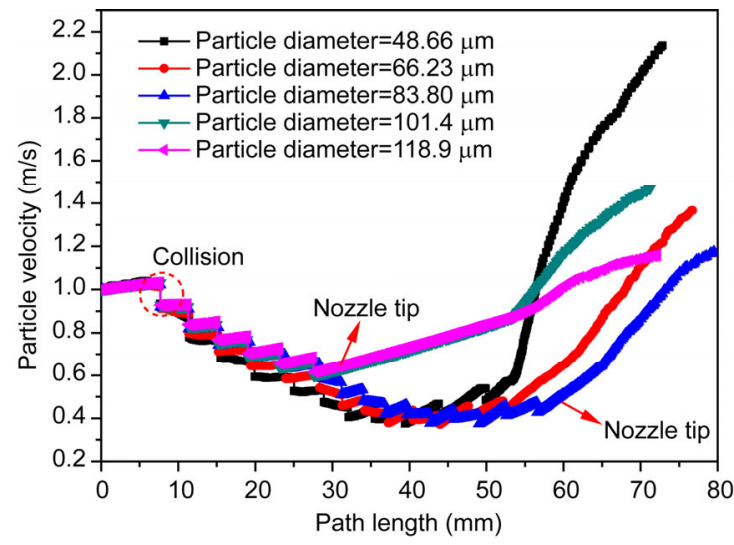

Figure 11 (Color online) Velocity curves of particle trajectories with different diameters.

diameter and restitution coefficient are two major factors of the collision behavior and particle velocity in the coaxial nozzle. The particle diameter is associated with the time of collision with the internal wall. When the diameter is greater than $100 \mu \mathrm{m}$, the collision occurs 6 times and the velocity at the nozzle tip is in the range of $0.6 \mathrm{~m} / \mathrm{s}$ to $0.8 \mathrm{~m} / \mathrm{s}$. When the diameter is smaller than $100 \mu \mathrm{m}$, the number of collisions increases to 14 times and the velocity at the nozzle tip decreases to about $0.4 \mathrm{~m} / \mathrm{s}$. It can be seen that the collision leads to step change of particle velocity. The momentum loss of each collision is evaluated by the restitution coefficient. The restitution coefficient in this case is set to 0.9 , so the velocities of the particle with diameter of $83.80 \mu \mathrm{m}$ before and after the first collision are $1.02 \mathrm{~m} / \mathrm{s}$ and $0.92 \mathrm{~m} / \mathrm{s}$, respectively. The effects of particle diameter and restitution coefficient on the powder stream are discussed in follow sections.

\subsection{The effect of particle diameter}

The powder particles can be classified into different size ranges by the sieve. When the powder is sieved by 140 mesh, 200 mesh, 270 mesh and 325 mesh, the diameter range of powder is 106-150 $\mu \mathrm{m}, 75-106 \mu \mathrm{m}, 53-75 \mu \mathrm{m}$ and 45-53 $\mu \mathrm{m}$, respectively [14]. The powder concentration along the nozzle axis on various diameter ranges is illustrated in Figure 12. The powder feed rate is kept at a constant value of $4.2 \mathrm{~g} / \mathrm{min}$. As seen in Figure 12 , the maximum powder concentration ranges from $3.0 \mathrm{~kg} / \mathrm{m}^{3}$ to 5.6 $\mathrm{kg} / \mathrm{m}^{3}$ for the standoff distance from $6.4 \mathrm{~mm}$ to $13.6 \mathrm{~mm}$ under different diameter ranges. Apparently, the powder in the diameter range of 53-75 $\mu \mathrm{m}$ is ideal for the coaxial laser cladding under the given gas flow rates. The results indicate that the particle diameter can alter the powder stream characteristics. Thus, the powder should be sieved to an appropriate particle diameter range before laser cladding process in order to provide a high-quality deposition and high powder using efficiency.

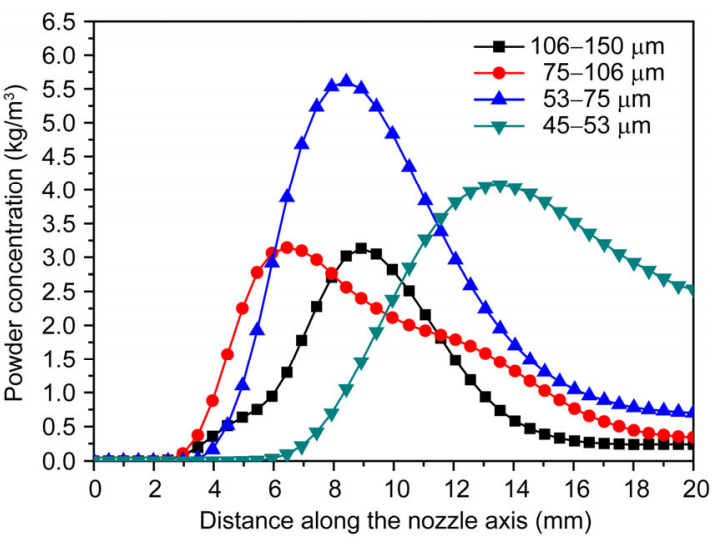

Figure 12 (Color online) Powder concentration along the nozzle axis on various diameter ranges.

\subsection{The effect of restitution coefficient}

Figure 13 shows the powder stream which is represented by 60 trajectories when the restitution coefficient is (a) 0.91 and (b) 0.99. All of the trajectories plotted are started from a same point of a nozzle inlet. Particles under smaller restitution coefficient have lower velocity when coming out of the nozzle tip as a result of the more momentum loss in collisions. It can be inferred that a lower velocity and smaller deviation angle can cause the trajectory of particles more close to the path line of carrying gas flow. Hence, the particle flight path below the nozzle tip is much concentrated.

The effect of restitution coefficient on the powder concentration distribution along the nozzle axis is presented in Figure 14. The powder particle diameter is in the range of $45 \mu \mathrm{m}$ to $130 \mu \mathrm{m}$ for all these simulations. The maximum powder concentration decreases from $3.23 \mathrm{~kg} / \mathrm{m}^{3}$ to 1.30 $\mathrm{kg} / \mathrm{m}^{3}$ and the powder focal distance decreases from 7.43 $\mathrm{mm}$ to $4.46 \mathrm{~mm}$ when the restitution coefficient increases from 0.91 to 0.99 . It can be seen that the powder concentration in the focus plane increases with the decrease of restitution coefficient. This result may be due to a decrease in the particle velocities at the nozzle tip. Hence, an appropriate choice of coaxial nozzle material is essential for the powder stream convergence.

\section{Conclusion}

A 3D numerical model considering the particle collision behavior at the nozzle interior wall was developed for the powder transporting process in coaxial laser cladding. The model was employed to investigate the powder stream structure, the collision and the relationship between them. According to the results, the following conclusions are derived.

(1) The developed model is able to predict the powder stream structure, and the simulation results are in accordance with the experimental results reasonably. The powder 
(a) Particle velocity

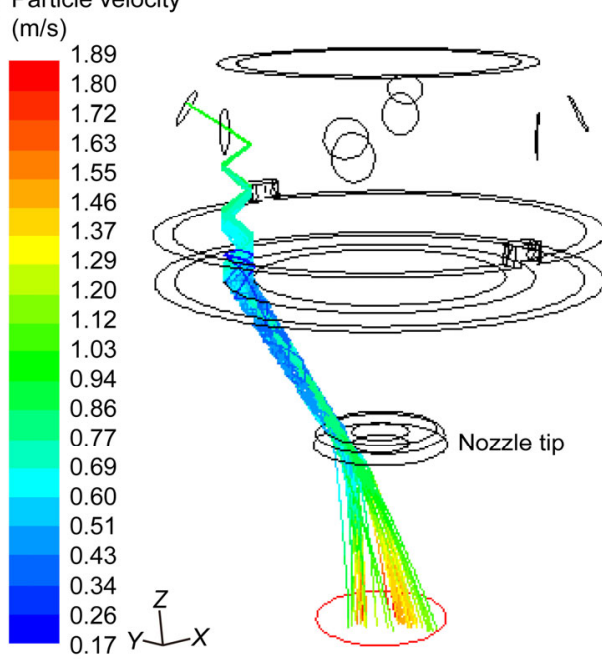

(b) Particle velocity

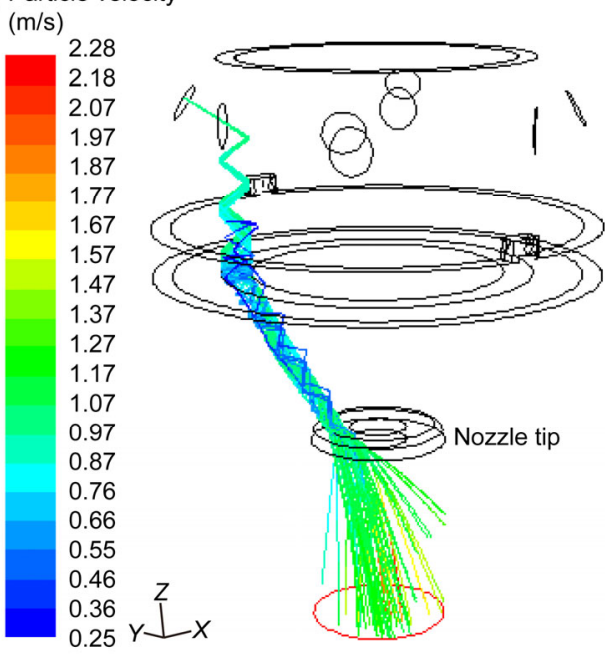

Figure 13 (Color online) Trajectories of particles when the restitution coefficient is (a) 0.91 and (b) 0.99 .

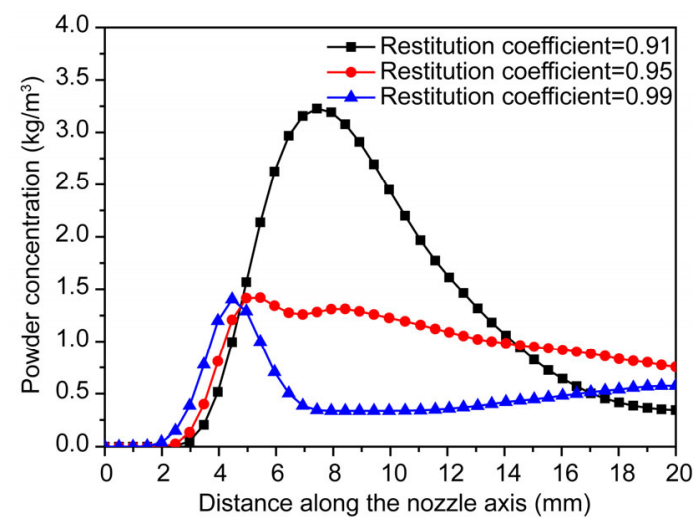

Figure 14 (Color online) Powder concentration with various restitution coefficients along nozzle axis.

stream below the nozzle tip exhibits a convergence shape that can be distinguished into three zones: annular zone, consolidation zone and dispersed zone. The desirable depo- sition position that the substrate should be placed is in the consolidation zone. When the gas flow rates are selected as constants, the convergence position of powder stream keeps invariable with varying powder feed rate, ensuring the stability of laser cladding process.

(2) In the powder transport process, particles collide with interior wall of the coaxial nozzle many times until they get out of the nozzle tip. The location and the flight direction of particles are dispersed when the particles reach the horizontal plane of the nozzle tip due to the collisions. The particle diameter and restitution coefficient significantly affect the collision behavior within the nozzle, subsequently leading to the change of powder concentration distribution below the nozzle.

(3) The particle diameter can alter the powder stream characteristics, offering an approach to optimize the powder stream by sieving the powder to certain diameter range for the coaxial laser cladding. The powder in the diameter range of 53-75 $\mu \mathrm{m}$ is ideal for the coaxial laser cladding under the given gas flow rates, which are $8.5 \mathrm{~L} / \mathrm{min}$ of inner shielding gas, $3 \mathrm{~L} / \mathrm{min}$ of carrying gas and $4 \mathrm{~L} / \mathrm{min}$ of outer shielding gas. The peak value of powder concentration with restitution coefficient of 0.91 is almost 2 times than that of 0.99 .

This work was supported by the National Natural Science Foundation of China (Grant Nos. 11272316 and 11272317), and a project funded by the Priority Academic Program Development of Jiangsu Higher Education Institutions (PAPD).

1 Majumdar J D, Manna I. Laser material processing. Int Mater Rev, 2011, 56: 341-388

2 Dubourg L, Archambeault J. Technological and scientific landscape of laser cladding process in 2007. Surf Coat Tech, 2008, 202: 5863-5869

3 Li S X, Yu G, Zhang J C, et al. Single-row laser beam with energy strengthened ends for continuous scanning laser surface hardening of large metal components. Sci China-Phys Mech Astron, 2013, 56: 1074-1078

4 Toyserkani E, Khajepour A, Corbin S. Laser Cladding. Boca Raton: CRC Press, 2005. 1-40

5 Smurov I, Doubenskaia M, Zaitsev A, Comprehensive analysis of laser cladding by means of optical diagnostics and numerical simulation. Surf Coat Tech, 2013, 220: 112-121

6 Pinkerton A J, Li L. Modeling powder concentration distribution from a coaxial deposition nozzle for laser-based rapid tooling. J Manuf Sci, 2004, 126: 33-41

7 Pinkerton A J. An analytical model of beam attenuation and powder heating during coaxial laser direct metal deposition. J Phys D-Appl Phys, 2007, 40: 7323-7334

8 Liu J C, Li L J. Effects of powder concentration distribution on fabrication of thin-wall parts in coaxial laser cladding. Opt Laser Technol, 2005, 37: 287-292

9 Yang N. Concentration model based on movement model of powder flow in coaxial laser cladding. Opt Laser Technol, 2009, 41: 94-98

10 Lin J. Numerical simulation of the focused powder streams in coaxial laser cladding. J Mater Process Tech, 2000, 105: 17-23

11 Zhu G X, Li D C, Zhang A F, et al. Numerical simulation of metallic powder flow in a coaxial nozzle in laser direct metal deposition. Opt Laser Technol, 2011, 43: 106-113 
12 Zekovic S, Dwivedi R, Kovacevic R. Numerical simulation and experimental investigation of gas-powder flow from radially symmetrical nozzles in laser-based direct metal deposition. Int J Mach Tool Manu, 2007, 47: 112-123

13 Tabernero I, Lamikiz A, Ukar E, et al. Numerical simulation and experimental validation of powder flux distribution in coaxial laser cladding. J Mater Process Tech, 2010, 210: 2125-2134

14 Wen S Y, Shin Y C, Murthy J Y, et al. Modeling of coaxial powder flow for the laser direct deposition process. Int J Heat Mass Tran, 2009, 52: 5867-5877
15 Balu P, Leggett P, Kovacevic R. Parametric study on a coaxial multi-material powder flow in laser-based powder deposition process. J Mater Process Tech, 2012, 212: 1598-1610

16 Haider A, Levenspiel O. Drag coefficient and terminal velocity of spherical and nonspherical particles. Powder Technol, 1989, 58: 63-70

17 Du W, Bao X, Xu J, et al. Computational fluid dynamics (CFD) modeling of spouted bed: Influence of frictional stress, maximum packing limit and coefficient of restitution of particles. Chem Eng Sci, 2006, 61: 4558-4570 